\title{
En la cima del patrocinio deportivo \\ Análisis de un caso con altas cotas de éxito en el nuevo contexto digital
}

On Top of Sports Sponsorship

A Case-Study with High Levels of Success at the New Digital Context

Francisco Cabezuelo Lorenzo

Universidad de Valladolid-Campus de Segovia cabezuelo@hmca.uva.es

Juan Enrique Gonzálvez Vallés

Universidad San Pablo CEU y Universidad Complutense de Madrid

juanenrique.gonzalvezvalles@ceu.es

Mónica Viñarás Abad

Universidad San Pablo CEU

monica.vinarasabad@ceu.es
Cabezuelo Lorenzo, F. , Gonzálvez Vallés J.E., Viñarás Abad, M. (2015), En la cima del patrocinio deportivo: análisis de un caso con altas cotas de éxito en el nuevo contexto digital

Revista Internacional de Investigación en Comunicación aDResearch ESIC. No 12 Vol 12

Segundo semestre, Julio-Diciembre 2015 · Págs. 8 a 27 DOI: 12.7263/ADRESIC.012.001 
Clasificación JEL: L1, M15, M31

Palabras clave:

Patrocinio Deportivo, Comunicación

Corporativa, Redes Sociales.

La gestión de la comunicación y el patrocinio deportivo son áreas de interés creciente. Partido de esta realidad, este trabajo intenta analizar y estudiar un caso reciente de éxito centrado en la alianza entre un banco internacional con origen español y un reconocido montañero experimentado. Para ello, analiza al completo la pertinente campaña de comunicación y reputación centrada en el uso de redes sociales como Facebook, Twitter y Youtube. Este trabajo estima, que junto con las tradicionales estrategias de comunicación, en el actual contexto de la sociedad digital, es indispensable el uso de las redes sociales como herramientas para interconectar e interactuar con los grupos de interés de las organizaciones empresariales con el fin de establecer una auténtica comunicación bidireccional. De este modo, este artículo pretender aportar algo de luz en esta línea de investigación desde la perspectiva académica y empresarial a través de su análisis, ejemplos y conclusiones expuestas obtenidas tras el estudio de las redes sociales usadas en este caso de éxito coprotagonizado por una entidad bancaria española. Para ello, empieza con una aproximación epistemológica al concepto de patrocinio deportivo actual, repasando algunos ejemplos. Finalmente, concluye con la afirmación de que el patrocinio deportivo puede ser una herramienta de comunicación extremadamente útil si se sabe adaptar a la nueva sociedad digital.

\section{ABSTRACT}

JEL Classification:

L1, M15, M31

Key words:

Sports Sponsorship,

Corporate Communication,

Social Media.
Communication management and sport endorsement are increasing areas of interest. Starting from this benchmark, this paper tries to analyze and study a successful recent case focused on the alliance between an international bank based in Spain and a well-known senior mountaineer. It analyses the complete communication and reputation campaign based on social media, including Facebook, Twitter and Youtube. This paper considers that, in the context of the current digital society, together with the traditional communication strategy, the social media have become indispensable platforms to interconnect and interact with groups of interest of each business organization that searches for a real two-way communication. Thereby, this paper pretends to be an enlightening study about the aims to achieve and the proposals on this academic and research line of interest, expounding conclusions and examples obtained after the analysis of social media campaign developed by a bank organization. We analyze general characteristics of sponsorship, endorsement management over sponsored view, and the management and relation concerning of the sponsoring firms demands. First of all, this study defines sponsorship concept and its characteristics. Therefore, it discusses mistaken conceptions about sport sponsorship use, and finally it deals management and sponsorship use as competitive and advantage communication tool in this contemporary digital society. 


\section{Introducción y justificación}

Las nuevas tecnologías se han ido incorporando a nuestra vida. Se han convertido el algo cotidiano para el ciudadano. Parece cada vez más evidente que en estos comienzos del siglo XXI estamos asistiendo a una profunda transformación en la forma en la que compartimos nuestra información. La digitalización de la información y la convergencia de este fenómeno con el de su distribución en red han traído consigo una nueva era del desarrollo humano de dimensiones históricas (Sotelo González, 2012: 217). Estos cambios suponen modificaciones en el modo de comunicación de todos los individuos, tanto a nivel personal como corporativo (Cabezuelo Lorenzo y Ruiz Carreras, 2010: 340). Las redes sociales están cambiando las relaciones de consumo entre empresas y usuarios. Así, los grandes anunciantes están apostando por los medios sociales y el éxito de sus estrategias en estos medios radica en la oferta de servicios de valor añadido y promociones exclusivas para los usuarios de sus microsites (Abuín Vences, 2014).

Las redes sociales han encontrado en el deporte un ámbito perfecto para su propagación. Se trata de un efecto que también se producido en el sentido contrario, puesto que el deporte se ha percatado de las grandes capacidades de las redes sociales para transmitir su mensaje, acercarse al ciudadano medio (Gonzálvez Vallés, 2012: 104). Para algunos investigadores como Hibai López González (2014: 13), los medios de comunicación han provocado en el deporte moderno profesional un proceso de «comodificación» (estandarización y acomodo) que en las últimas décadas ha convertido al deporte en un producto apto para el consumo de audiencias sociales.
El patrocinio en general ya ha sido tratado como herramienta de responsabilidad social por varios autores como L. F. Solano Santos (2009) y como herramienta de aproximación a públicos y stakeholders por otros como M. Viñarás Abad, J. Ma Herranz de la Casa y F. Cabezuelo Lorenzo (2013). De hecho, también hay literatura académica reciente sobre el fenómeno del patrocinio deportivo, como son los escritos de L. F. Solano Santos (2013) y M. Walraven, R. H. Koning, R. H. \& M. Van Bottenburg (2012). Cada vez también hay más trabajo sobre deporte y redes sociales. El deporte ha encontrado en las redes sociales un medio neutro, alejado de las tendencias de los grandes medios pertenecientes a los grandes grupos empresariales. Así, no es extraño que los deportes mayoritarios y las estrellas de los mismos hayan puesto sus ojos sobre las redes sociales. Aquí han encontrado un canal para transmitir su mensaje sin filtros de ningún tipo ni interpretaciones previas. Desde el pedestal donde están subidos, empresas, instituciones y líderes sociales pueden mostrarse cercanos a sus seguidores alrededor de todo el mensaje, construyendo un ecosistema favorable para sus intereses. De este modo, las grandes empresas han utilizado estas plataformas para mostrar a través de acciones de patrocinio y mecenazgo, su sensibilidad social. Como afirma María Teresa García Nieto (2012), atribuir a las empresas la cualidad de ejercer responsabilidades sociales supone conferir a éstas la capacidad de asumir las consecuencias que puedan tener sus acciones sobre los diferentes grupos sociales. El ejercicio de estas responsabilidades va a depender, sin embargo, de la naturaleza de las actividades que lleve a cabo cada organización y de los públicos implicados en ellas. 
Lo más sorprendente ha sido como los deportes minoritarios han encontrado también su hueco y, sobre todo, una mayor repercusión de su actividad. Raro es el club de fútbol de división humilde, club de patinaje, petanca o dominó que no posee su propio grupo de Facebook, cuenta de Twitter o perfil de Google Plus. El marketing relacional ha ayudado a que, al menos, se constituyan pequeños grupos o grupúsculos de familiares y amigos que tengan noticias actualizadas sobre estos equipos que antes desarrollaban su actividad en la más estricta intimidad. Sin embargo, el fenómeno que vamos a estudiar no encaja como caso típico en ninguno de los dos grupos anteriores. Es un caso híbrido que ayuda a crear un grupo nuevo. Cuando el banco BBVA comenzó a patrocinar al montañero español Carlos Soria gestó un proyecto para asociar su imagen a la de uno de los alpinistas más respetados, pero los resultados que consiguió estuvieron muy por encima de lo que podían esperar.

\section{Metodología y estructura}

El método expositivo y demostrativo es el más adecuado para el caso que nos ocupa, puesto que sirve para obtener conclusiones pertinentes. Resulta relevante conocer todos estos puntos puesto que solo así se puede entonces evaluar la sostenibilidad del proyecto y, en caso positivo, desgranar las posibles acciones futuras que se puedan llevar a cabo. Se busca, en definitiva, obtener los resultados positivos que, además, puedan ser aplicables a otros proyectos tanto dentro del mundo del deporte como en lo relacionado con otros patrocinios que ésta $u$ otras entidades deseen llevar a cabo.

Teniendo en cuenta la afirmación de $\mathrm{M}^{\mathrm{a}} \mathrm{Cruz}$ Alvarado López, Susana de Andrés del Campo y Rodrigo González Martín (2007: 204), que consideran que «la publicidad, dentro de la comunicación de masas, pasa por ser un torrente de mensajes normalmente infravalorados por el gran público por su obviedad y carácter banal», es fácil entender las razones que llevan a empresas e instituciones a buscar fórmulas alternativas, como es el caso del patrocinio y el mecenazgo. En este contexto, empresas e instituciones buscan campañas creativas de comunicación ajenas a la publicidad y basada en técnicas eficaces de «publicity» (Fanjul y Cabezuelo, 2013).

A pesar de que autores como J. Heijnen, M. de Reuver, H. Bouwman, M. Warnier y H. Horlings (2013) se centren en el estudio del contenido difundido en las redes sociales, como fórmula para medir el éxito de sus impactos, este trabajo analiza también aspectos cuantitativos como el número de seguidores o el incremento de los mismos. Para ello, se han revisado metodologías existentes como la propuesta desde Estados Unidos por la experta Nichole Kelly (2012) que desarrolla modelos para medir el retorno de la inversión tras la comunicación y promoción en las redes sociales. En este caso, se trata de una acción de patrocinio que busca mejorar en términos de imagen y que busca generar sinergias entre una organización y sus públicos, más allá de una acción comercial bancaria.

Así, el principal objetivo de este trabajo es descubrir las claves del éxito en el caso de la sinergia de éxito entre BBVA y Carlos Soria, a través del análisis de una de las herramientas más vivas de las relaciones públicas externas como es la técnica del patrocinio. Este caso de patrocinio deportivo ha provocado que se desate en las redes sociales todo un fenómeno singular e inigualable, sobre todo partiendo de la base de que el 
alpinismo es un deporte muy minoritario. Del mismo modo, este trabajo tiene presente la repercusión que se ha producido en los medios de comunicación, tanto tradicionales como digitales. Y, por supuesto, las conexiones o retroalimentación entre todos estos canales, puesto que los reclamos entre unos y otros han sido lo que han producido gran parte del éxito de este caso, sobre todo por la viralidad provocada.

Para ello, se realiza un análisis de los datos obtenidos tras el seguimiento pormenorizado de los perfiles de Carlos Soria en Facebook, Twitter y Youtube gracias al acceso directo a los datos facilitado por la organización y que se puede además contrastar con herramientas externas gratuitas y al alcance de cualquier investigador como son Edgerank Checker y Pagelever en el caso de Facebook y las herramientas de Topsy y de Twitter Counter en el caso de Twitter. Además, son datos y perfiles públicos. Para el uso de los gráficos además se ha usado el software de Microsoft Excel.

Este trabajo analiza pormenorizadamente la gestión de la comunicación, centrado en el estudio en las redes sociales. Al contar con tres perfiles activos, el análisis permite contemplar las diferencias fundamentales entre las diferentes herramientas digitales en aspectos o indicadores de importancia como el número de seguidores, el número de impactos o el número de publicaciones en cada uno de ellos. Está demostrado que «los entornos digitales aplacan la timidez que suele dificultar la participación de los alumnos en las aulas» según Evans y Matthew (2012), tal y como recogen Llorente, Bartolomé y Viñarás (2013: 645).

Esta metodología sirve para demostrar cómo funcionan individualmente cada uno de estos perfiles y su relación o relaciones con sus adep- tos, llámense fans, followers o suscriptores. Se parte de la base de que en todos los perfiles se intenta transmitir siempre de la mejor manera posible, con el objetivo último de crear una imagen positiva, de la cual se beneficien los dos actores de este patrocinio, que no son otros que el banco BBVA y el deportista Carlos Soria.

En primer lugar, este trabajo se basa en un amplio análisis de contenidos, poniendo el foco en el tipo y frecuencia de publicación de contenidos. Se trata de dos variables muy significativas para establecer las correspondientes conclusiones, puesto que permiten saber si tanto una como otra se están utilizando en el sentido de tratar de captar y/o fidelizar a los usuarios. En segundo lugar, y ligado estrechamente con lo anterior, este trabajo observa atentamente cuáles han sido las interacciones que se han realizado por parte de los usuarios en las diferentes publicaciones de los distintos perfiles. De este forma, es posible establecer también conclusiones sobre cuáles son los contenidos donde más interactúan los usuarios. Como valor añadido, y prestando especial atención a las particularidades de una red social como Facebook, este trabajo también logra analizar el nivel de «Me gusta» que recibe cada publicación. Se trata de una forma de interactuar muy particular, más sencilla y simple que cualquier tipo de comentario, y que globaliza la opinión de los usuarios. Constituirá, pues, un análisis independiente en nuestro estudio desde el punto de vista formal, pero integrado dentro del resto de análisis que establecerán las conclusiones finales, que se muestran divididas en generales y específicas.

\section{Análisis del caso y discusión}

La situación económica actual ha hecho que la imagen de las entidades bancarias se deteriore, al 
ser consideradas como las culpables de la misma. Resultando cierta o no esta afirmación, el lastre en términos de imagen para los bancos es patente. De hecho, podemos encontrar hasta encuestas que parten de organismos oficiales que dan fe de que los españoles ponen en su punto de mira a los bancos para buscar responsables de la actual situación económica. Ante esta realidad, real o artificial, las entidades bancarias buscan los mecanismos o herramientas que les sean más útiles para contrarrestar esta pérdida de imagen.

Dentro de las relaciones públicas externas, el patrocinio ha sido tradicionalmente uno de esos mecanismos que mejor resultado ha proporcionado. Es por ello que, al igual que otras entidades bancarias, BBVA ha ejercido desde siempre esta opción y han sido muy conocidas sus acciones de patrocinio en programas como la Ruta Quetzal BBVA, sus acciones de apoyo al mundo del arte en todo España a través de diferentes museos (Museo Nacional del Prado, Fundación Joan Miró de Barcelona, Museo Guggenheim de Bilbao, la Fundación Albéniz y Escuela Superior de Música Reina Sofía) y su apoyo a la lengua y cultura española a través de la Fundación del Español Urgente (Fundéu BBVA). Del mismo modo, ha puesto en marcha varias campañas de apoyo a los jóvenes como Blue BBVA y de patrocinio local y cercano, sobre todo en el País Vasco, donde mantiene su sede social.

Pero, además de todas estas acciones, BBVA se ha centrado sobre todo en un elemento con connotaciones tan positivas como es el mundo del deporte. Sus principales apuestas han venido de los deportes mayoritarios y de las estrellas que en ellos militan. Así, en acuerdo con la Liga de Fútbol Profesional, BBVA pasó a ser el principal patrocinador de la Liga española, que pasó a llamarse Liga BBVA en su Primera División; y Liga Adelante, en su Segunda División. En este caso se referencia la marca a la máxima categoría del fútbol español, mientras que el eslogan principal de la entidad queda como reclamo o claim del segundo escalón.

En este mismo sentido, BBVA buscó unos embajadores de marca mundiales que también poseyeran una reconocida e irrefutable buena imagen con la que poder asociarse. La lista quedó acotada por encuestas y consultas que permitían escoger a los preferidos por los españoles, entre los que destacan estrellas del deporte. Habiendo referenciado ya su imagen a la del fútbol español, BBVA decidió nombrar como embajadores de marca a Andrés Iniesta y a Iker Casillas. Dos jóvenes deportistas, muy valorados por la gente y pertenecientes a los dos equipos grandes del fútbol español, Barcelona y Real Madrid, Real Madrid y Barcelona.

Además de sus grandes acciones en el mundo del fútbol, BBVA se ha convertido también en el banco oficial de la National Basketball Association, la famosa NBA de Estados Unidos. Esta segunda gran apuesta deportiva del banco de origen vasco pero con presencia global poseía un valor estratégico muy importante. BBVA llegó a un acuerdo con la NBA para el ser el banco oficial de la prestigiosa liga de baloncesto estadounidense. No se ejercía un patrocinio con una presencia tan importante como en el caso de Liga BBVA, pero se implantaba la marca BBVA para un entorno de influencia claro y amplio, puesto que abarcaba desde los propios Estados Unidos, donde BBVA está presente, hasta su repercusión en los países sudamericanos, donde la NBA es seguida e idolatrada, incluso más que la liga de fútbol española. 


\subsection{La alianza entre BBVA y Carlos Soria}

En este contexto de grandes estrellas y magnos eventos deportivos, sin embargo, destaca el deseo de BBVA de apostar también por un deporte tan minoritario como es el alpinismo y por un personaje mucho menos conocido que las estrellas de la NBA y la liga española de fútbol. Así, surge la colaboración con el alpinista español Carlos Soria Fontán. A finales de agosto de 2011, BBVA y Carlos Soria establecen una alianza estratégica por la cual la entidad bancaria colaborará con el alpinista español mediante la fórmula del patrocinio para su objetivo de lograr coronar las catorce montañas más altas de la tierra. Se trata de una apuesta por un deportista con valores como la tenacidad, el esfuerzo, la superación y la humildad a través de la madurez de un septuagenario montañero. Soria ya había conquistado once ochomiles y BBVA se comprometía a financiar sus próximas expediciones para tratar de hollar las cimas de los tres retos pendientes en la agenda del alpinista español: el Annapurna, el Kanchenjunga y el Dhaulagiri. Nacido en Ávila el 5 de febrero de 1939, es uno de los alpinistas españoles con mayor prestigio. Es la persona de más edad en lograr la cima en las catorce montañas más altas del mundo. Carlos Soria es la única persona en el mundo que ha escalado nueve ocho-miles con más de sesenta años, y tres de ellos con más de setenta. Carlos Soria se ha convertido en un referente para todos aquellos que consideran que la edad no debe convertirse en un obstáculo para cumplir todos los objetivos que te propongas. Su tenacidad y espíritu de superación han inspirado a muchas personas para disfrutar de una edad avanzada pero sin complejo y con ganas de disfrutar al máximo de todo lo que pueda. El historial de
Carlos Soria se remonta a cuando, con catorce años, realizó su primera excursión a la Sierra de Guadarrama en Madrid. Desde ahí, sus viajes han sido casi innumerables, desde 1968, cuando formó parte de la primera expedición española que viajó a Rusia para subir el monte Elbrus, hasta 1973, cuando también formó parte de la primera expedición española que viajó al Himalaya para tratar de coronar el Manaslu, hecho que él no conseguiría hasta 37 años después, en 2010.

La mayoría de las ocasiones en que Carlos Soria ha estado de expedición en el Himalaya lo ha hecho solo, acompañado únicamente de algún sherpa y/o algún porteador. Desde que cuenta con el patrocinio de BBVA, Soria lidera su propia expedición, con la presencia de un médico, cámaras y varios sherpas y porteadores que le ayudan en la consecución de su objetivo. De este modo, Carlos Soria se ha convertido en una de las personalidades más respetadas y queridas del mundo del deporte en general, y del alpinismo en particular. Su espíritu humilde y sus grandes retos y hazañas son más propios de espectadores del deporte que de un actor protagonista. Esta asociación sin duda beneficiaba a los dos actores principales del patrocinio pero había que elevar al gran público esta idea para que tuviera el grado de conocimiento y aceptación adecuados.

\subsection{Revisión cuantitativa del caso de Carlos Soria y su presencia en las redes sociales}

Con el fin de lograr una difusión adecuada a los nuevos tiempos y retos digitales, BBVA puso su empeño en las redes sociales para llevar a cabo esta labor, focalizando su esfuerzo en Facebook, Twitter y YouTube. 


\subsubsection{Facebook}

Sin duda, la más exitosa de las redes sociales, por lo menos en el caso de BBVA-Carlos Soria. A pesar de que el alpinista poseía una cuenta personal ${ }^{1}$ se creó una página como personaje público ${ }^{2}$ con mucho éxito desde su génesis a finales de agosto de 2011 .

A continuación, y como se hará con los perfile de Twitter y YouTube, se exponen los datos recogidos durante un año de seguimiento al que ya se puede calificar como «fenómeno Carlos Soria». En primer lugar, el histórico de los seguidores en Facebook del perfil creado por BBVA,
La red social que creó Mark Zuckerberg fue la que más esfuerzo recibió por parte del patrocinador, subordinando los otros perfiles a ésta primera. Fruto de esta estrategia llegaron los increíbles resultados que se registraron en cuanto a número de seguidores. Pronto se pasó de no tener ninguno a superar la extraordinaria barrera de los 200.000. Como nuestro estudio se centró en el primer año de actividad en las redes sociales, la cifra que encontramos al final de este periodo resultó asombrosa, prácticamente tocando los 233.000 fans de la página «Yo Subo con Carlos Soria».

\section{Gráfico 1 - Evolución histórica de los seguidores «Yo Subo con Carlos Soria»}

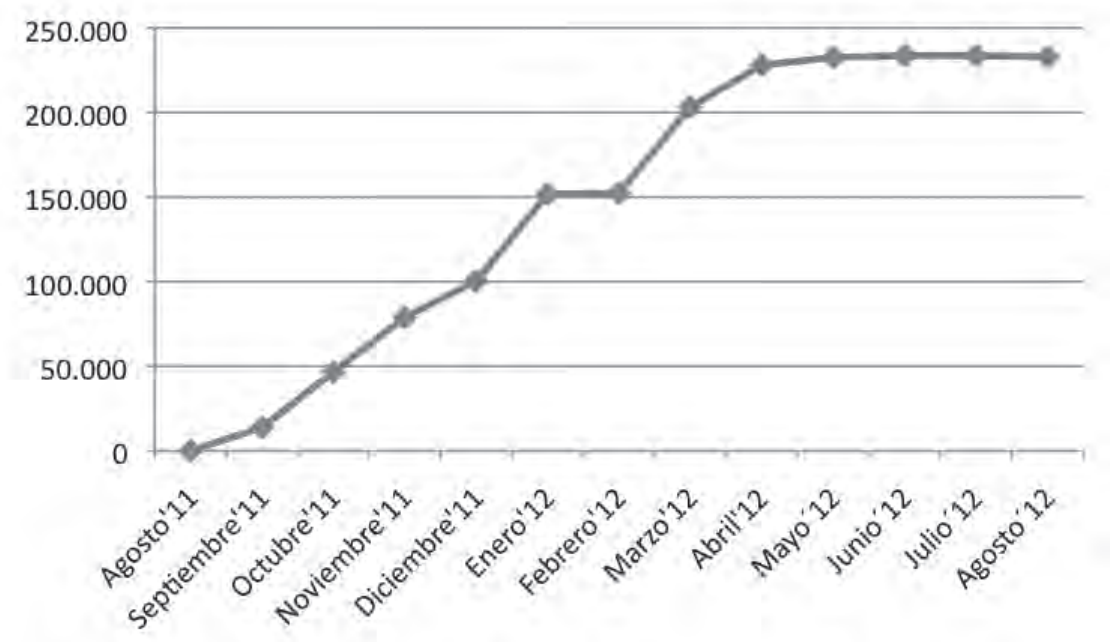

Fuente: Facebook.

que ha marcado un hito en cuanto al seguimiento de personajes públicos se refiere.

1 http://www.facebook.com/carlos.soriafontan

2 http://www.facebook.com/yosuboconcarlossoria
El análisis de este gráfico nos lleva a utilizar la palabra impresionante en primer lugar. En menos de seis meses, "Yo Subo con Carlos Soria» había logrado más de 100.000 seguidores, algo al alcance de pocos perfiles de personajes conoci- 
dos. Tenemos en cuenta que, entre medias, Carlos Soria y sus compañeros acometieron una primera expedición, ya bajo el paraguas de BBVA, tratando de coronar el Dhaulagiri. Este hecho, que se produjo fundamentalmente entre los meses de septiembre y octubre de 2011, produjo sin duda un efecto llamada ante la cantidad y calidad de contenidos aportados en dicho perfil.

El segundo punto fuerte del análisis de este histórico llega en un periodo que podemos considerar como 'valle', puesto que entre noviembre y marzo no existe ninguna expedición por parte de Carlos Soria al Himalaya. Sin embargo, la continua actualización del perfil con nuevos contenidos, unido a la presencia de Carlos Soria en medios de comunicación, estrategia complementaria pero sin duda muy efectiva, logró que los seguidores continuaran incrementándose de forma espectacular.

El tercer aumento importante de seguidores se produjo durante la expedición de Carlos Soria al Annapurna. Considerado como normal, los seguidores aumentaron al volver a producirse el momento clímax que todos los seguidores estaban esperando, fidelizando a los actuales y aumentando la viralidad que produjo un mayor número de nuevas incorporaciones. Es lógico que, al ofrecer de nuevo contenidos de alta calidad y, sobre todo, totalmente diferenciados del casi el 100\% de otros personajes públicos, exceptuando otros alpinistas, se produjera este incremento que dejó los seguidores superando los 233.000 en el mes de junio de 2012. A partir de ese momento se ha producido un estancamiento en el número de seguidores de este perfil en Facebook. Queda saber, quizá en un análisis posterior, si los dígitos han llegado ya a su tope y es difícil aumentarlos o una nueva expedición sería capaz de aumentarlos todavía más.

\subsubsection{Twitter}

El principal damnificado del esfuerzo hecho en Facebook, o mejor dicho para Facebook, fue el perfil de Twitter, con un éxito sólo relativo. Al igual que ocurría con Facebook, Carlos Soria ya poseía una cuenta personal en Twitter (@carlossfontan) pero BBVA se decidió a crear un perfil oficial (@RetoCarlosSoria) para contar todas las novedades sobre el alpinista y sus expediciones. Los contenidos seguían los que se publicaban en Facebook y la mayoría de ellos se referenciaban a esta red social.

El principal problema con el que se ha encontrado este perfil es el de no poder anotar un seguimiento más continuo que fueran más en la línea de lo que esta red social consume. Al poseer una temporalidad más alta, puesto que en los timelines de los perfiles de los usuarios de Twitter los mensajes aparecen y son sustituidos por otros nuevos con más frecuencia, la no repetición de los mensajes o la creación de mensajes novedosos hacen perder atractivo a esta cuenta. Aun así, el aumento ha sido sostenido durante estos primeros doce meses, destacando un fuerte aumento durante la expedición primaveral al Annapurna, con más de 600 nuevos seguidores o followers. Los números son discretos, pero el perfil en Twitter ya cuenta con más de 3.500 followers y en ningún mes se ha producido un descenso de seguidores, con lo que se espera que dentro del segundo año puedan alcanzar números significativos.

\subsubsection{YouTube}

El principal contenedor de vídeos del mundo ha sido utilizado más como apoyo de los otros dos perfiles y, sobre todo, de Facebook, que como otra red social en busca de nuevo seguidores. Los suscriptores no han sido demasiado numerosos, tal y como lo reflejan las estadísticas recopiladas. 


\section{Gráfico 2 - Evolución histórica de los seguidores «@RetoCarlosSoria»}

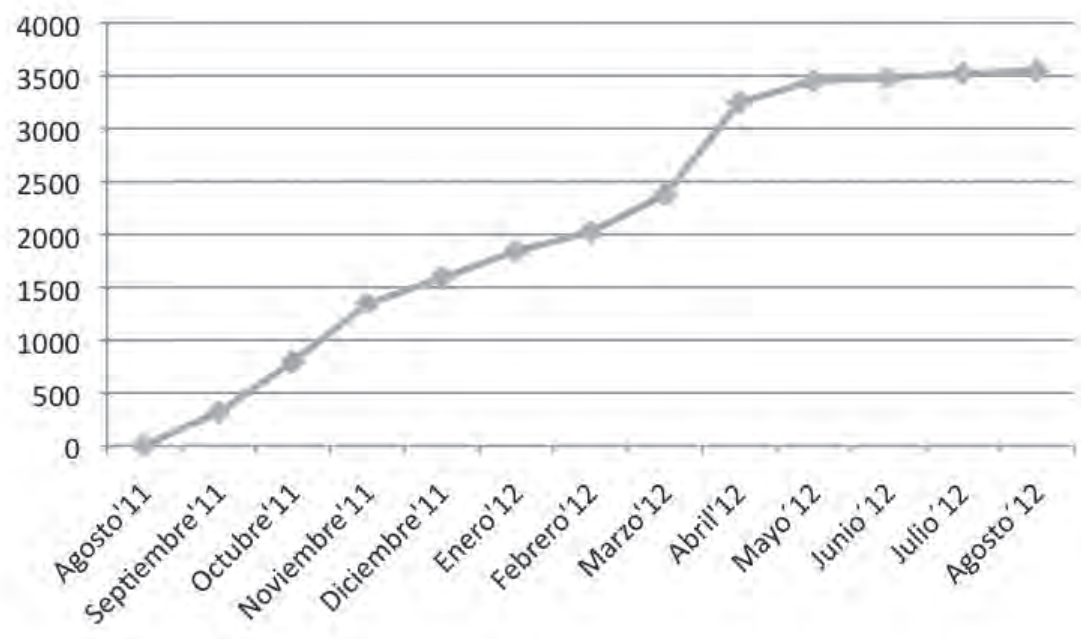

Fuente: Twitter.

\section{Gráfico 3 - Evolución histórica de los seguidores «Yo Subo con Carlos Soria»}

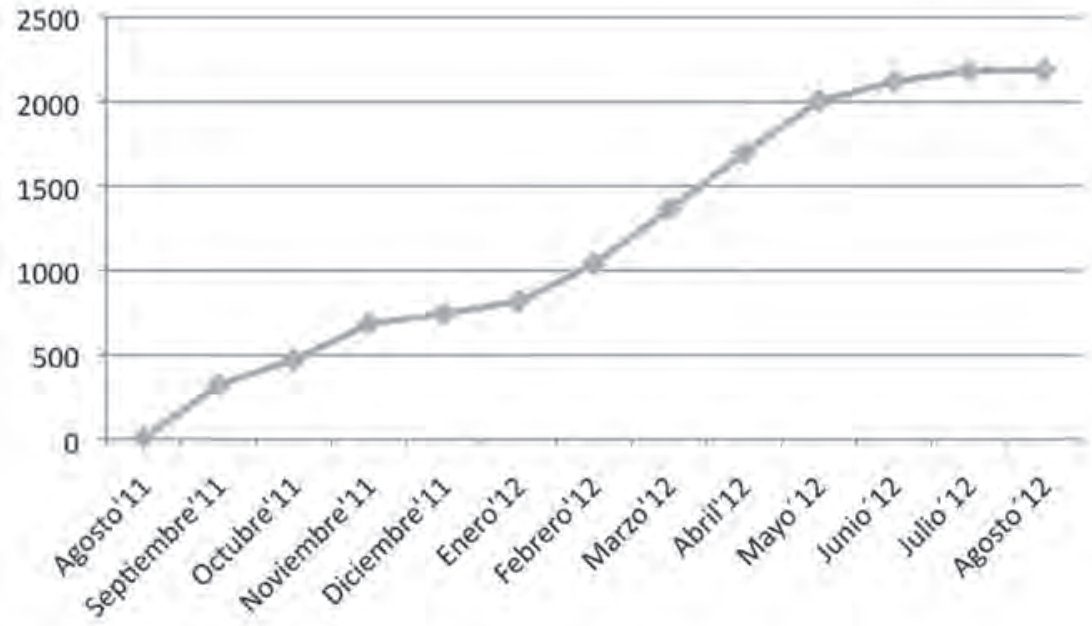


El número de suscriptores es poco significativo pero, sin embargo, queda para un estudio posterior la importancia que los vídeos proporcionados por este canal han tenido en el éxito global de este patrocinio. Simplemente apuntaremos que hasta agosto de 2012 se habían superado las 300 piezas audiovisuales, superando las 900.000 reproducciones. Un pequeño apunte de lo beneficioso que ha sido YouTube para la estrategia de comunicación de patrocinio ejercida por BBVA.

\subsubsection{Comparativa de redes sociales}

En una comparativa entre Facebook, Twitter y YouTube obviamente resalta la diferencia en el número de usuarios, es decir, los fans, followers, seguidores y suscriptores de los distintos perfiles, que «suben» con Carlos Soria.

\subsection{Análisis de contenidos}

\subsubsection{Consideraciones previas}

Los dos parámetros más significativos a la hora de evaluar la comunicación en los perfiles de las redes sociales de BBVA-Carlos Soria son la tipología y la frecuencia. En esta parte del trabajo se exponen cuáles son los diferentes tipos de contenidos que se han realizado y en qué periodos se ha publicado más y/o menos. Sin embargo, antes de avanzar, y a tenor de los resultados obtenidos en la primera parte de nuestro estudio, es preciso hacer una consideración. Se observa la red social de más éxito fue sin duda Facebook, con más de 230.000 fans captados dentro de su primer año de existencia.

Además, se observa también como Twitter fue utilizado como un simple replicante de los con-

\section{Gráfico 4 - Comparativa usuarios redes sociales BBVA-Carlos Soria}

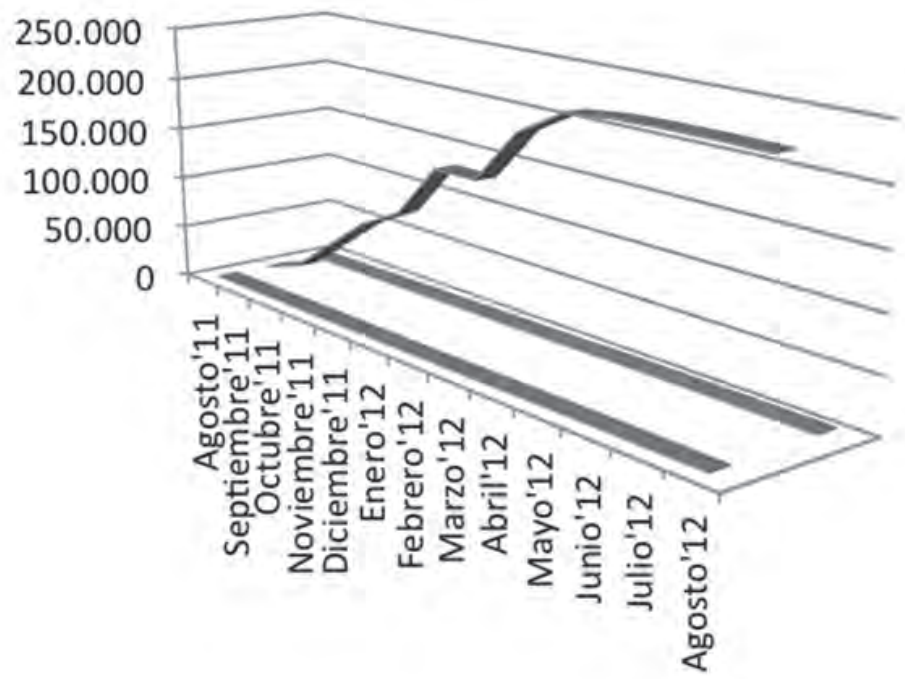

YouTube

Facebook

- Twitter 
tenidos de Facebook, sin aportar nada nuevo ni especial; y como YouTube se constituyó en la plataforma perfecta para aglutinar las distintas piezas audiovisuales que se realizaron.

Por todo ello, y para no entrar en análisis superfluos o incluso carentes de sentido, hemos optado por centrar nuestro foco en el perfil de Facebook, sin lugar a dudas el más paradigmático y el que nos va a permitir realizar un análisis más profuso de los contenidos. De hecho, no hay ningún contenido publicado en Twitter o YouTube que no haya sido publicado también en Facebook y, sin embargo, no todo el contenido publicado en Facebook ha sido publicado en las otras dos.

\subsubsection{Tipología}

Es preciso detallar cuáles son los tipos de publicación que se han dado dentro del perfil de Facebook «Yo Subo con Carlos Soria». En total son siete:
1. Actualización de estado: Textos sin otro tipo de elemento auditivo, visual o audiovisual.

2. Compartir: historias de otros usuarios que se comparten en el muro.

3. Enlace: una URL procedente de otra página web que se incrusta en el estado.

4. Foto: bien sea individual o pueden ser varias mediante la creación de una galería fotográfica.

5. Música: pieza sólo de audio que se inserta en el muro.

6. Pregunta: cuestiones a modo de encuesta que se publican con varias opciones de respuesta para que el usuario elija una de ellas.

7. Vídeo: también se inserta en el estado o se puede subir directamente al modo de una fotografía.

En total, se registraron 769 publicaciones en el muro de «Yo Subo con Carlos Soria», donde las fotos y los vídeos fueron los más numerosos:

\section{Gráfico 5 • Número de publicaciones según tipología en “Yo Subo con Carlos Soria”}

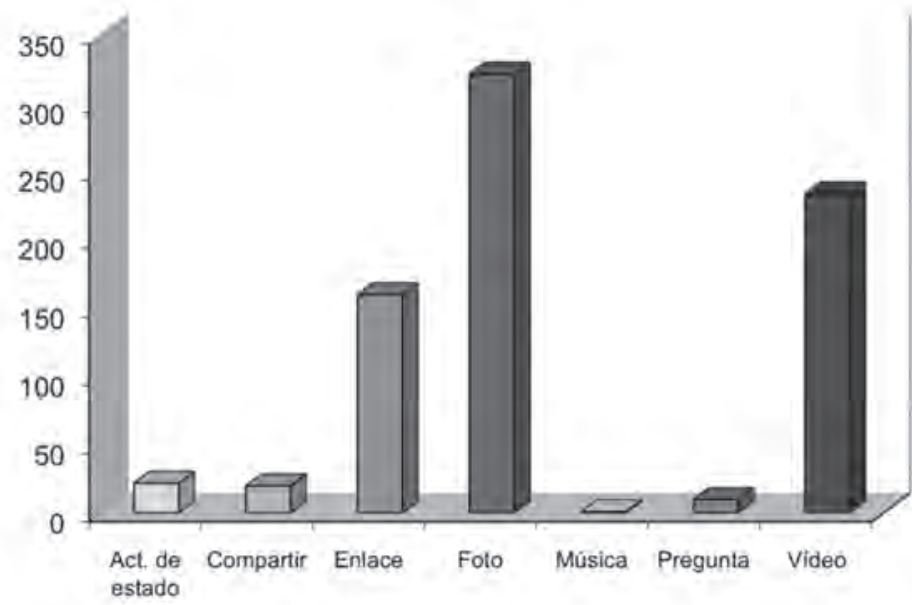

Fuente: Elaboración propia a partir de los datos de Facebook. 
A raíz de los resultados mostrados en el gráfico, observamos como los elementos audiovisuales y los gráficos han sido los que más veces se han reproducido en «Yo Subo con Carlos Soria». También resulta relevante observar como los enlaces externos también han poseído una gran importancia alcanzando la cifra de 160 publicaciones. Por último, casi se pueden considerar residuales el resto de publicaciones que, en el mejor de los casos, superan por poco la veintena de resultados.

\subsubsection{Frecuencia}

A la hora de compararla frecuencia de publicación se pretende obtener una visión de conjunto sobre las publicaciones puesto que, además, en el caso de Carlos Soria existen periodos bien diferenciados de mayor atención, como es el caso de las expediciones a los 'ochomiles'. Deberemos observar, por tanto, cuál es la frecuencia de las publicaciones en general y también según la tipología de las mismas. (Gráficos 6 y 7)

En primer lugar, se observa un claro pico en cuanto al número de publicaciones en el periodo correspondiente a la expedición al Annapurna, en los meses de marzo, abril y mayo de 2012. Sin embargo, y quizá por el estado embrionario del proyecto, no se produce un aumento significativo en el periodo de la expedición al Dhaulagiri, que corresponde a los meses de septiembre y octubre de 2011.

Además, se constata el aumento de la publicación de fotografías en los periodos de expedición, tanto en la del Dhaulagiri como en la del Annapurna, aunque en ésta última el aumento es mucho más explícito. Igualmente, destacamos por el lado contrario la casi nula publicación de elementos musicales, las actualizaciones de estado y la publicación de preguntas, consti- tuyendo elementos estadísticamente despreciables para nuestro análisis.

\subsection{Interactividad}

Tras la revisión de estos gráficos es lógico preguntarse si existe una correspondencia entre la tipología y la frecuencia de los contenidos publicados con el grado de interactividad de los usuarios. Se pretende observar si este canal presenta paradojas como que un elemento con escaso número de publicaciones, como por ejemplo una pregunta, puede poseer sin embargo un alto impacto entre los fans de «Yo Subo con Carlos Soria». O si, por el contrario, existe una correlación entre los elementos más publicados, vídeos y fotos, con los 'Me gusta', los comentarios o las veces que un usuario ha compartido este contenido en su muro.

Para ello es preciso recurrir de nuevo a tres tipos de gráficos que nos aporten una explicación global a esta hipótesis. En primer lugar, un sumatorio total de las respuestas que han recibido las publicaciones de forma mensual, con lo que obtendremos la correlación, o no, en la frecuencia de las publicaciones y su respuesta entre los usuarios. Se establece así ahora una observación en cuanto a la categoría de pregunta, puesto que, al no recibir ni 'Me gusta' ni comentarios, asimilaremos las respuestas como si de comentarios se tratara. Es una forma justa de medir esta interacción y que permite homogeneizar todo los tipos de contenidos.

En segundo lugar, se observa también de forma gráfica cuál es la tipología de las respuestas que se han recibido de forma mensual, con lo es posible resaltar de qué forman interactúan más los usuarios en relación con la frecuencia e intensidad de las publicaciones que se hayan realizado. Por último, es necesario obtener respuesta 
Gráfico 6 • Número de publicaciones por meses en “Yo Subo con Carlos Soria”

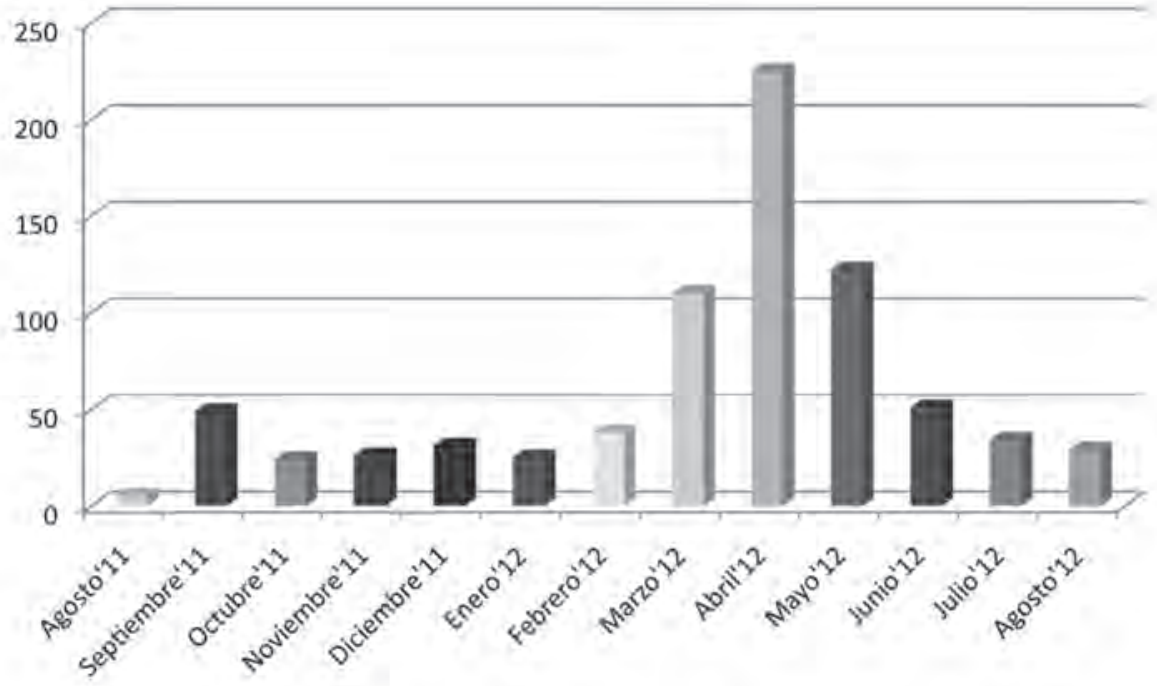

Fuente: Elaboración propia a partir de los datos de Facebook.

\section{Gráfico 7 • Número de publicaciones por meses y según tipología}

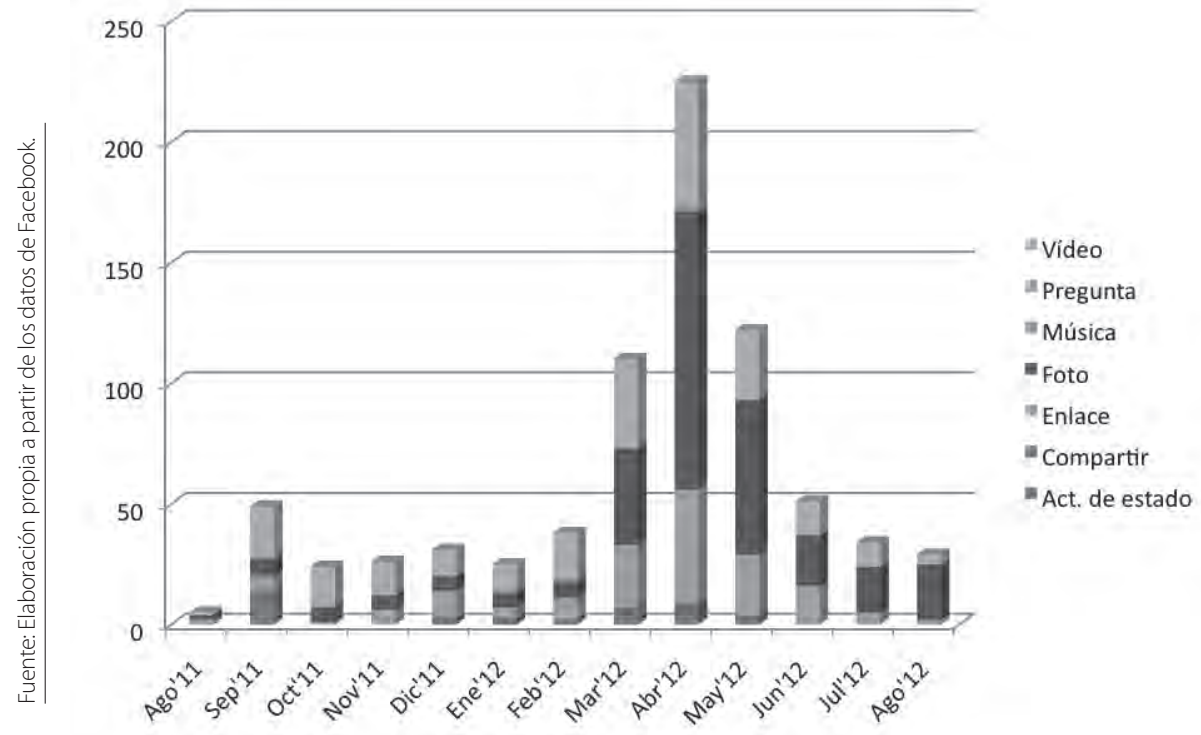


para la relación pura entre el tipo de publicación que se haya utilizado en el perfil y la interactuación de los usuarios. (Gráficos 8, 9 y 10)

Después de observar estos gráficos podemos mostrar la correlación entre las interacciones y las publicaciones del canal «Yo Subo con Carlos Soria». Primero observamos que el periodo de expedición al Annapurna fue el que, sin duda, obtuvo mayor respuesta entre los usuarios. Además, también destacan los «Me gusta» sobre el resto de interacciones posibles dentro de los contenidos. En último lugar, podemos apuntar una clara relación entre los contenidos fotográficos y el mayor grado de interacción. Son este tipo de publicaciones, seguidas de los vídeos y los enlaces, los que obtienen una mejor respues- ta por parte de los fans del alpinista de 74 años en Facebook.

Finalmente, es preciso apuntar una interesante reflexión sobre las interacciones en «Yo Subo con Carlos Soria». Evaluamos todas ellas como positivas, es decir, que no valoran negativamente las publicaciones, ni, por supuesto, con faltas de respeto o insultos. Somos conscientes de que, como en todos los perfiles de este tipo, existe uno o varios moderadores que filtran los comentarios aparecidos en las publicaciones y purgan los que no tengan un sentido totalmente positivo. Además, los «Me gusta» son un tipo de comentario o interacción especial, cerrada y que sólo pretenden reflejar la positividad de la misma por parte del usuario.

\section{Gráfico 8 • Número de interacciones por meses en "Yo Subo con Carlos Soria”}

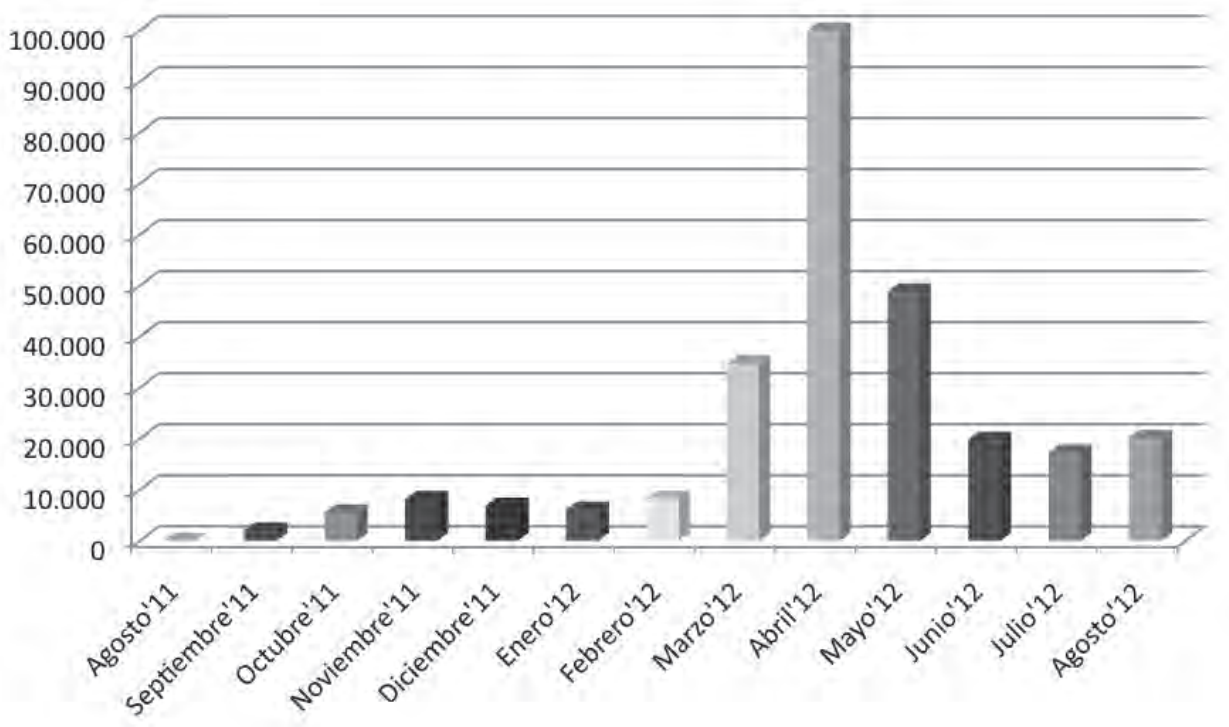




\section{Gráfico 9 • Número de interacciones por meses y según tipología}

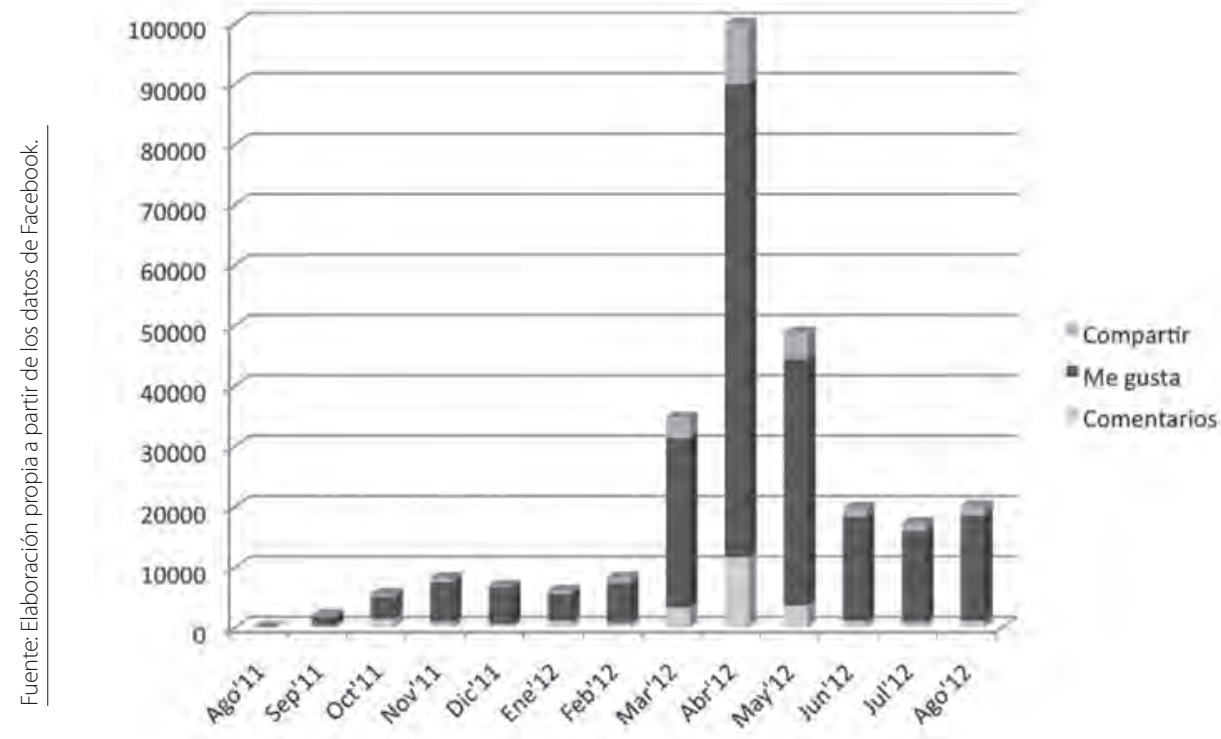

\section{Gráfico 10 - Número de interacciones por tipos y por tipo de publicación}

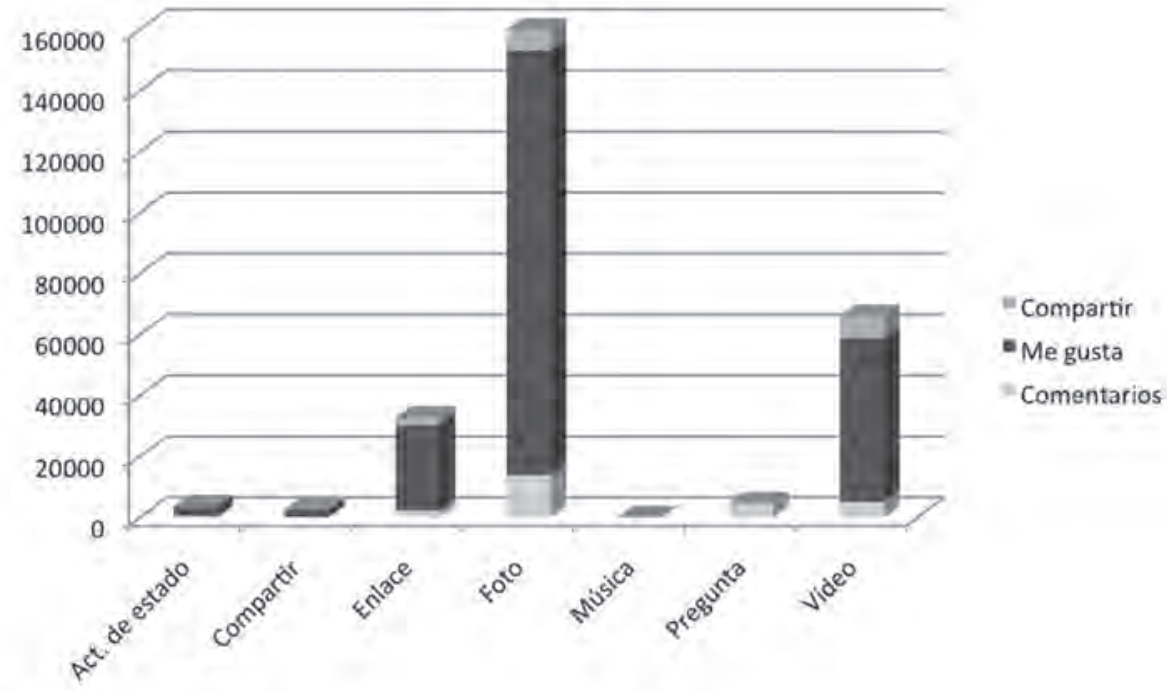

Fuente: Elaboración propia a partir de los datos de Facebook. 


\section{Conclusiones}

\subsection{Conclusiones generales}

Los resultados de esta investigación ponen de manifiesto que la estrategia de patrocinio de BBVA-Carlos Soria en redes sociales ha sido un acierto y exitosa de todo punto. Hemos constatado como la entidad bancaria poseía un problema de imagen, fruto de la crisis económica actual. Como los españoles creen que los bancos son parte del problema, más que de la solución para el estado actual del país. Fruto de ello fue la búsqueda de un patrocinio que llevara asociados valores positivos y que encajara con la política de patrocinios de BBVA, focalizada en el deporte. En este sentido, el acuerdo con la Liga de Fútbol Profesional para patrocinar lo que pasaría a llamarse la Liga BBVA; y el acuerdo con la NBA para ser el banco, resultaron excelentes bancos de pruebas para buscar otro acuerdo en el mismo campo. Carlos Soria ha resultado ser, no sólo una apuesta ganadora, sino que ha pasado a convertirse en todo un símbolo que lo liga a los valores BBVA, que lo elevó a la categoría de embajador mundial de su marca. No es que por este patrocinio se haya revertido la imagen que BBVA tenía pero el interés demostrado por el alto número de seguidores sí que supone un espacio de promoción perfecto para transmitir lo valores que la entidad bancaria desea sobre sí misma. Con casi 250.000 seguidores, aunando los de los tres perfiles de Facebook, Twitter y YouTube, Carlos Soria se ha convertido en un fenómeno mediático muy por encima de cualquier expectativa inicial, y superando a muchos de los más afamados deportistas españoles, y parte también de los extranjeros. En este punto, queda por analizar el origen geográfico de los seguidores, que será objeto de análisis de un artículo de investigación posterior. El mayor esfuerzo en las redes sociales se ha producido, sin lugar a dudas, en Facebook. La inclusión de contenidos que se adaptan a las características de esta red social han supuesto un boom que llevó al perfil «Yo Subo con Carlos Soria» a superar los 230.000 seguidores en menos de un año.

\subsection{Conclusiones específicas}

El esfuerzo, sin duda, transcurrido este primer año será el de fidelizar esta cantidad ingente de personas. El resto de perfiles han tenido un éxito menor, puesto que no cabe calificar el número de seguidores de Twitter y de YouTube como un fracaso. En el caso de Twitter, nunca ha habido intención de adaptar los mensajes a las características de esta red social, con lo que resulta lógico que el interés despertado sea menor. La mejoría vendría también con un seguimiento más detallado de los periodos de expediciones, que aumente la viralidad de este perfil. En el caso de YouTube, los suscriptores son escasos pero, sin embargo, las reproducciones de los vídeos han sido todo un éxito. Esto indica que la labor de apoyo de YouTube a los perfiles de Facebook y Twitter, sobre todo el primero, sí que ha sido fructífera. Sólo podemos apuntar una posible mejoría en esta red social a través de la mayor calidad del formato de los vídeos, que podría suponer un incremento no sólo de suscriptores, sino también de seguidores en otras redes sociales. Atendiendo a la tipología de las publicaciones del canal «Yo Subo con Carlos Soria» en Facebook, existe una clara preferencia por los elementos gráficos, fotos y galerías, y los audiovisuales, vídeos. También existe una clara correspondencia entre los dos elementos anteriores, tipología y frecuencia, con el grado de interactividad de los usuarios. Son muchos más los comentarios, los «Me gusta» y las veces que 
se ha compartido el contenido en los periodos de expediciones, que en el resto de los primeros doce meses que forman parte de nuestro estudio. Esta correlación de los tres elementos apunta al acierto de BBVA-Carlos Soria en la publicación de contenidos, siendo una de las claves de su éxito. Los «Me gusta» son las interacciones más usadas, seguidas por los comentarios, ya a gran distancia, y por último la posibilidad de compartir el contenido publicado en el muro del usuario. En este caso los fans de Facebook han encontrado una forma rápida y cómoda, aunque cerrada y sin posibilidad creativa alguna, de mostrar su simpatía y empatía con respecto a un contenido. Además, hay una clara relación directa entre la tipología de los contenidos y la respuesta de los usuarios. Es cierto que las fotos y los vídeos son los tipos que reúnen un mayor número de publicaciones pero, además, son los más comentados, los más compartidos y los que más «Me gusta» acumula. En cuanto a interactividad destacamos esta relación directa entre fotos y vídeos como ejemplo más destacado y, sin embargo, también resalta el caso de los enlaces que obtienen un nivel de interactividad mucho menor con respecto al nivel de publicaciones, que es bastante alto. El resto de publicaciones, actualizaciones de estado, compartir, música y pregunta, vuelven a tener una relación directa entre número de publicaciones y respuesta de los usuarios, siendo bajo en ambos casos.

Las conclusiones de esta investigación, a pasar de estar basadas en datos puntuales de unas fechas concretas, no se han quedado obsoletas. Por el contrario, tienen vigencia y la contribución de este artículo al ámbito de las comunicaciones corporativas puede ser de gran utilidad para el mundo profesional y académico, sobre todo desde la perspectiva de cómo las marcas que asocian su imagen a deportistas, pueden mantener conversaciones con sus públicos a través de los medios sociales, basadas en la transmisión de los valores del deportista a la marca.

Entre los retos abiertos que deja esta investigación se presenta también la posible discusión, que se deja para un futuro, de plantear la necesidad de buscar indicadores que permitan monitorizar las acciones que realizamos para alcanzar nuestros objetivos (conocidos como KPIs) en nuestras campañas en las redes sociales, temas ya propuestos por otros autores como Heijnen et alt (2013) y Kelly (2012). 


\section{Bibliografía}

Abuín Vences, N. (2014). Las estrategias publicitarias de los anunciantes españoles en los Social Media. El caso de Facebook. Revista Internacional de Investigación en Comunicación aDResearch ESIC. ISSN 1889-7304, No 9 Vol 9. Primer semestre, enero-junio 2014. Págs. 64-75.

Alvarado López, M. C., De Andrés del Campo, S. \& González Martín, R. (2007): Discapacidad: estigma y concienciación. Comunicación e Cidadanía: Revista Internacional de Xornalismo Social $=$ Social Journalism International Review, ISSN 1886-8975, Núm. 1. Págs. 203-222.

Cabezuelo Lorenzo, F. y Ruiz Carreras, M. (2010). Comunicación digital y política en Aragón. Una fórmula para la bidireccionalidad en la interacción entre políticos y ciudadanos. Revista Latina de Comunicación Social, 65. La Laguna (Tenerife): Universidad de La Laguna, páginas 340 a 353. DOI: 10.4185/RLCS-65-2010-904-340-353

Evans, R. y Matthew, A. (2012). «Should we still lecture? Reconsidering pedagogical approaches to promote student engagement, challenging the traditional lecture». En: INTED 2012, Conference technical program. Recuperado [15-102013] de http://eprints.qut.edu.au/49062/2/49062.pdf

Fanjul Peyró, C. y Cabezuelo Lorenzo, F. (2013). How to get your message across: Últimas tendencias creativas y procesos de innovación en campañas de comunicación. Madrid: Editorial Fórum XXI. 153 págs. ISBN: 978-84-616-7400-8.

García Nieto, M. T. (2012). Las Ciencias Sociales y la RSC. Revista Internacional de Investigación en Comunicación aDResearch ESIC. ISSN 1889-7304, No 6, 2012 (Ejemplar dedicado a: Responsabilidad Social Corporativa). Págs. 92-111. Gonzálvez Vallés, J. E. (2012). El reto de Carlos Soria (I). La creación de un fenómeno en redes sociales. Revista de Comunicación Vivat Academia. ISSN: 1575-2844. Diciembre de 2012. Año XV. No 121. Págs. 103-116. http://dx.doi. org/10.15178/va.2012.121.103-116

Gonzálvez Vallés, J. E. (2013). El reto de Carlos Soria (II). Contenidos e interactividad. Revista de Comunicación Vivat Academia. ISSN: 1575-2844. Marzo de 2013. Año XV. No 122. Págs. 106--116. http://dx.doi.org/10.15178/va.2013. 122.106- 120
Heijnen, J., de Reuver, M., Bouwman, H., Warnier, M., \& Horlings, H. (2013). «Social media data relevant for measuring key performance indicators? A content analysis approach.» (Págs. 74-84). Co-created effective, agile, and trusted eServices. (15th International Conference on Electronic Commerce, ICEC 2013, Turku, Finland). Springer Books, Heildelberg. ISBN: 978-3-642-39807-0

López González, H. (2014). El rol de los medios en la trans formación del deporte en bien de consumo. Doxa Comunicación: revista interdisciplinar de estudios de comunicación y ciencias sociales. ISSN 1696-019X. No XVIII. Págs. 13-28.

Llorente Barroso, C., Bartolomé Muñoz de Luna, Á. y Viñarás Abad, M. (2013). Implementación del aprendizaje basado en problemas (ABP) y el learning by doing en el Grado en Publicidad y RRPP para la adquisición de competencias. Historia y Comunicación Social. No Extra 18. Vol. 2. Págs. 639650. http://dx.doi.org/10.5209/rev_HICS.2013.v18.44264

Kelly, N. (2012). How to measure social media: A step-by-step guide to developing and assessing social media ROI. Que Publishing-Pearson Education, Indianápolis, Estados Unidos. ISBN-10: 0789749858. Págs. 288.

Solano Santos, L. F. (2009): Patrocinio y mecenazgo: instrumentos de responsabilidad social corporativa. Editorial Fragua, Madrid. ISBN-13: 9788470742729. Págs. 208.

Solano Santos, L. F. (2013): Responsabilidad Social Corporativa en el Patrocinio Deportivo. Historia y Comunicación Social. Vol. 18. Número especial de octubre. Págs. 255-265. http://dx.doi.org/10.5209/rev_HICS.2013.v18.43960

Sotelo González, J. (2012). Deporte y social media: el caso de la Primera División del fútbol español. Historia y Comunicación Social. Vol. 17. Págs. 217-230. http://dx.doi.org/ 10.5209/rev_HICS.2012.v17.40607

Viñarás Abad, M., Herranz de la Casa, J. Mª \& Cabezuelo Lorenzo, F. (2013). La gestión cultural y del ocio como estrategia de relación con los públicos. Revista Internacional de Relaciones Públicas, 3 (6). Universidad de Málaga. Págs. 177-196. Walraven, M., Koning, R. H. \& Van Bottenburg, M. (2012): «The Effects of Sport Sponsorship: A Review and Research Agenda». The Marketing Review. Vol. 12. No 1. Spring 2012. Westbum Publishers Ltd. Págs. 17-38. 


\section{Recursos electrónicos}

http://www.youtube.com/yosuconcarlossoria

http://www.facebook.com/yosuboconcarlossoria

http://www.twitter.com/retocarlossoria

http://prensa.bbva.com/informacion-corporativa/patroci-

nios/\%2825-9905\%29.html

https://ngo.bbvasuma.com/user/bbva 